\title{
Differentiated welding deposit of soiling parts according to abrasive wear map
}

\author{
Viktor Myalenko, and Olga Sankina* \\ Federal State Educational Institution of Higher Education "Kuzbass State Agricultural Academy", \\ Markovtseva st., 5, 650056 Kemerovo, Russia
}

\begin{abstract}
The paper proposes the use of maps of the expected abrasive wear of the friction surface of a soil-cutting part, which characterize the wear intensity at various points of the friction surface during its movement in the soil environment. The importance of this work is caused by changes in modern technologies for the cultivation of agricultural crops, requiring changes in the geometric shapes of soil-cutting parts with the need to predict their service life. A study of the physical and mechanical properties and structure of the hardened layer was carried out using gray cast iron. Gray cast iron was preliminarily subjected to heat treatment, which made it possible to increase the tensile strength up to $200 \ldots 250 \mathrm{MPa}$ and hardness up to HRC $50 \ldots 55$. To increase the wear resistance of soil-cutting parts operating in an abrasive environment, hardening was carried out by the electric spark method using gray cast iron, previously subjected to heat treatment. The microstructure of the deposited layer became pearliteledeburite without graphite precipitation. Electro-spark deposition made it possible to form a wear-resistant layer in several passes, while the structure of the cast iron and the base did not undergo any changes. No increase in grain size was observed in the deposited layer, which favorably affects its properties. The hardness of the deposited layer was higher (4800 .. 5000 $\mathrm{MPa})$ than that of the base metal (2300 ... $2400 \mathrm{MPa})$, no softening of the base was observed. According to the test results of hardened products, heat-treated gray cast iron can be recommended for use as a material for hardening, since the thickness of the coating does not lead to changes in the initial geometric dimensions of the soil-cutting tool using differentiated surfacing using abrasive wear maps.
\end{abstract}

\section{Introduction}

Modern materials and technologies used to strengthen the blades of soil-cutting parts operating in an abrasive soil environment make it possible to increase their service life to varying degrees while maintaining the quality of technological operations. The very fact of the loss of the working state of the soil cutting parts is a natural consequence of their wear when working in an abrasive environment. Solid particles of the parent rock, such as quartz, granite, feldspar, etc., found in the soil environment, abrade the friction surfaces of the soilcutting parts. When the soil-cutting parts of the plowshares, plowshares, various teeth and

\footnotetext{
* Corresponding author: olga_vk_06@mail.ru
} 
knives are worn out, they change their initial parameters, thereby reducing productivity and increasing energy costs. The range of materials currently used for hardening the blades of soil-cutting parts is very diverse [1].

The choice of one or another material and the hardening technology itself occurs, as a rule, on the basis of an analysis of the ratio of the price of the material and the expected service life of the part. At the same time, when discarding worn-out soil-cutting parts, a significant amount of unrealized surfacing materials remains on their friction surfaces. According to our observations on different designs of rejected parts, the amount of unrealized surfacing material is about $10-40 \%$ [2].

These aspects set the urgent task of the modern search for a rational (differentiated) placement of surfacing materials on the friction surfaces of soil-cutting parts, which makes it possible to increase the resource of the part and ensure the expedient consumption of surfacing materials. To solve the above problem, a certain sequence of actions is required to ensure differentiation of the distribution of strengthening materials over the friction surface. This search is based on the need to know the expected rates of wear at different points of the soil-cutting parts. That is, a certain graphic model or map of the expected intensity of abrasive wear of the surface of the part is needed and, taking into account the data of such a map, apply a hardening material.

The purpose of this work is to find a method for the differentiated deposition of surfacing material on the friction surface of damaged parts according to the initial map of the abrasive wear intensity.

\section{Materials and methods}

To achieve this goal and build an initial map of abrasive wear, we used imitation loading of soil-cutting parts on laboratory benches for accelerated tests [3]. Figure 1 shows a general view of the bench equipment.

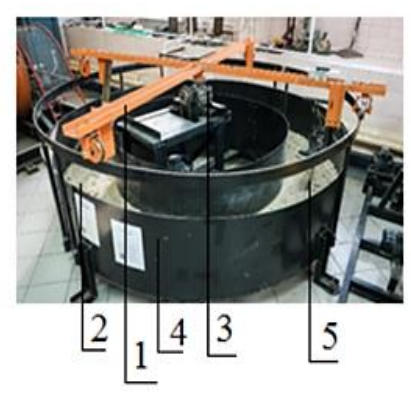

a

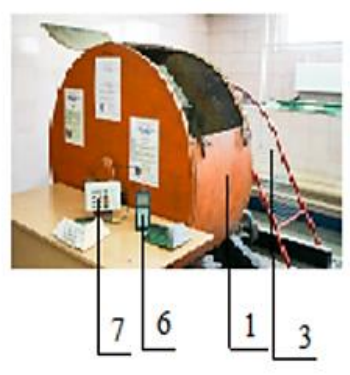

$\mathrm{b}$

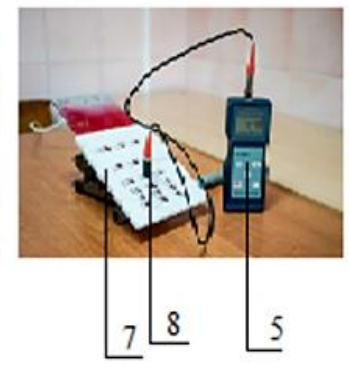

c

a) test-bench for accelerated tests with a horizontal axis of rotation; b) the same test-bench but with a vertical axis of rotation; c) digital thickness gauge with conductor;

1 - body, 2 - soil medium, 3 - drive of the rotation shaft, 4 - carrier gear, 5 - working body with the tested soil-cutting part, 6 - digital thickness gauge, 7 - jig plate, 8 - feel gauge.

Fig. 1. General view of test-bench equipment.

The soil medium 2 was placed inside the body 1 . By means of the shaft drive 3 and the carrier gauge 4 , the working body was moved with the tested soil-cutting part 5 in the soil medium 2. The point values of the wear of the easily abraded material were measured with a digital thickness gauge 6 using the jig plate 7 and the feel gauge 8 . 


\section{Research results and discussions}

The abrasive wear map was built for the friction surface of the soil-cutting part of the ripper. Parts with different loosening angles of $20^{\circ}$ and $45^{\circ}$ were tested. An easy-to-wear material was applied to the friction surface of the soil-cutting part of the ripper tooth, and the thickness of the wear layer at different points was measured with a digital thickness gauge. The values of the normal pressures were measured from the Amonton-Coulomb law.

$$
N=\frac{\Delta И 3}{\operatorname{tg} \varphi \cdot K}
$$

where: $\mathrm{N}$ - normal pressure; $\Delta$ wear is the point value of the measured material wear from the friction surface; $\varphi$ - angle of friction of the soil against the surface of the part; $\mathrm{K}$ a coefficient characterizing the resistance to abrasive wear of the friction surface material (in this case, for an easily abraded layer).

The $\Delta$ wear values obtained from the research results were presented in the form of maps of the intensities of abrasive wear of two samples of soil-cutting parts with different loosening angles of $20^{\circ}$ and $45^{\circ}$.

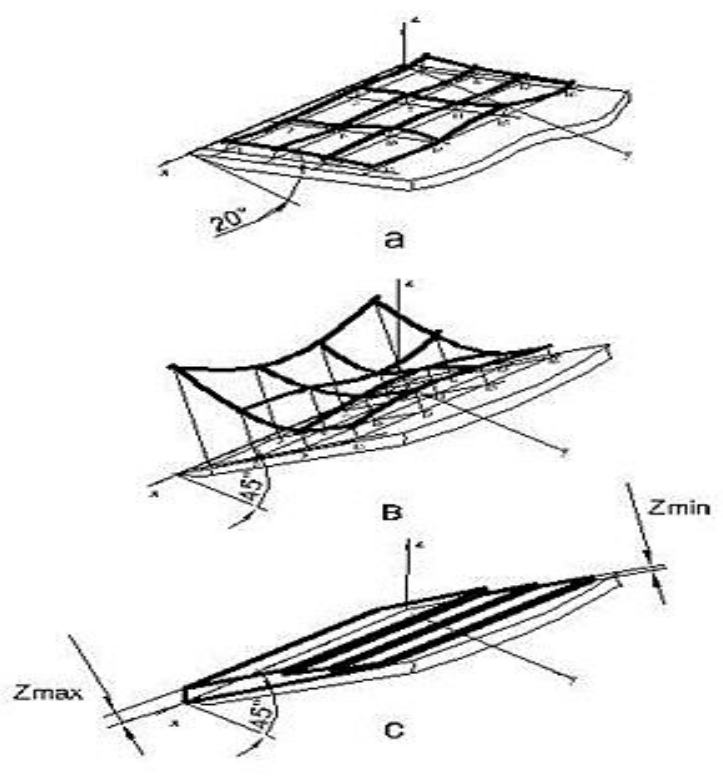

a) angle of loosening $20^{\circ}$; в) angle of loosening $45^{\circ}$; c) application of wear-resistant material

Fig. 2. Maps of abrasive wear of the friction surface of the soil-cutting part.

For the wear map (Fig. 2, c) at an angle of loosening of $45^{\circ}$, for example, an analytical expression of a curved surface is given below, limiting the vertices of the vectors $\Delta$ wear.

$$
\begin{gathered}
\Delta \text { wear }\left(Z_{45}\right)=30,7+0,27 y+0,91 x-0,03 y^{2}-0,03 x^{2}+0,000 y^{3}+0,0002 x^{3}-0,2 x y \\
+0,004 x^{2}+0,003 y^{2}
\end{gathered}
$$

Where $\mathrm{x}, \mathrm{y}$ - are the coordinates of the application of wear rate vectors on the friction surface. 
Equation (2) can be analyzed analytically or a section can be considered separately according to the wear intensity map in the ZOX planes (Fig. 2, a, c). Then, for the contours of different sections, we have the following regression equations:

$$
\begin{aligned}
& Z_{10}=35,93+8,06 x+1,47 x^{2}, \\
& Z_{20}=16,03-0,46 x-0,32 x^{2}, \\
& Z_{30}=6,28-2,01 x+0,63 x^{2}, \\
& Z_{40}=2,03+0,59 x-0,08 x^{2} .
\end{aligned}
$$

Where $Z_{10}, Z_{20}, Z_{30}, Z_{40}$ - are functions of the ordinates $Z$ spaced from the edge of the blade at distances of 10, 20, 30 and $40 \mathrm{~mm}$, respectively. Then equations (2) and (3) are the initial data for the technology of applying a hardening layer of wear-resistant materials on the blade part of the soil-cutting element, for example, as shown in Figure 2, c.

The thickness of the deposing layer of the wear-resistant material is selected according to the conditions of further operation and the mechanics of contact interaction / abrasive particles, where the following components are required, such as the assessment of soils by wear capacity, the initial material of the soil-cutting part and the properties of the hardening layer of wear-resistant materials [4].

Today, all soils are divided into three conditional categories according to their wearing capacity. The first category has wear capacity of $1.3 \ldots 3.0 \mathrm{~g} / \mathrm{ha}$, the second $-0.5 \ldots 1.3$ $\mathrm{g} /$ ha, the third $-0.37 \ldots 0.65 \mathrm{~g} / \mathrm{ha}$ [5]. Then, ultimately, the thickness of the deposited layer is determined taking into account the peculiarities of the subsequent operation of the soilcutting parts.

Highly alloyed hard alloys containing expensive alloying elements are usually used as wear-resistant coatings [6-15]. Typically, the hardening process is carried out by applying a wear-resistant material to those parts that are subject to more intense wear. The formation of a wear-resistant layer is associated with a number of difficulties, such as a change in the structure of the base material and the applied layer, as well as a change in the mechanical properties of both the base and the deposited metal. Wear-resistant materials are applied to the substrate in various ways as electrospark alloying, electric arc surfacing, powder coating of wear-resistant materials, and others.

To implement a layer of wear-resistant material differentiated in thickness in accordance with the obtained maps of abrasive wear, the most expedient is the method of electrospark alloying. Currently, electrodes in the form of rods and mixtures of various powders are most often used as wear-resistant coatings: sormite, relite, stalinite, high-alloy steel, cast iron.

Indeed, when using different materials and technologies and when applying hardening coatings, there is also the likelihood of quality risks due to the absence of an adhesion zone of the hardened layer with the base one, overheating of the base material, the formation of fragile particles in the adhesion zone and at the base, and others [6,7].

The transition zone plays a decisive role in a high-quality hardened layer. When analyzing compounds that are obtained using high-alloy cast iron (sormite), such zone is absent (Figure 3). 


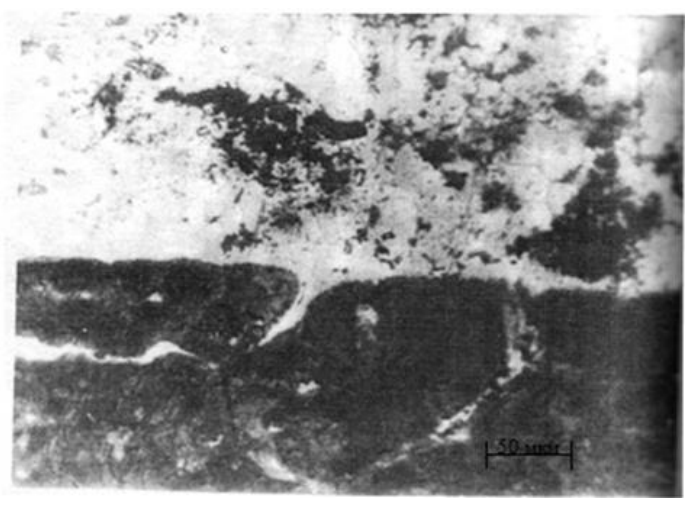

Fig. 3. Microstructure of composition steel $65 \mathrm{G}$ - sormite 1, deposited by electric arc method (x260).

Electric arc surfacing of hardening materials makes it possible to obtain a layer of any thickness, but this method has another drawback. With this method of hardening, a strong heating of the base occurs in the adhesion zone, which leads to the formation of large grains and a decrease in strength characteristics.

To change the physical and mechanical properties in the adhesion zone and in the base material, it is recommended to use heat treatment, since it is the most accessible.

As a hardening material, there was used gray cast iron which was preliminarily quenched in the temperature range of $700 \ldots 1050{ }^{\circ} \mathrm{C}$, holding at these temperatures for 30 minutes, followed by cooling in water. The final heat treatment was tempering at $300{ }^{\circ} \mathrm{C}$.

Another method of heat treatment used for gray cast iron electrodes is cyclic annealing ( 2 cycles) at a temperature of $950{ }^{\circ} \mathrm{C}$ with exposure period of 1 hour, after which a redistribution of graphite inclusions and crushing of graphite into smaller particles was observed. The amount of graphite has decreased approximately two times from $19.3 \%$ to $7.2 \ldots 7.8 \%$.

After heat treatment, there was an increase in mechanical characteristics $\left(\sigma_{\mathrm{w}}=200 \ldots\right.$ $250 \mathrm{MPa}$ ) and a pearlite-ledeburite structure without the presence of graphite particles. After tempering at the indicated temperatures, an increase in the hardness of cast iron to HRC $50 \ldots 55$ was observed without the presence of hardening cracks, which is very important when applying a hardening layer. Therefore, gray cast iron is the optimal material for differentiated hardening of soil-cutting implements.

Differential hardening was carried out using electric arc and electric spark surfacing with electrodes that were subjected to heat treatment according to the specified scheme.

Preliminary heat treatment of gray cast iron electrodes made it possible to obtain a perlite-ledeburite structure (Figure 4) and a high-quality wear-resistant layer that allows the tools to work in an abrasive environment.
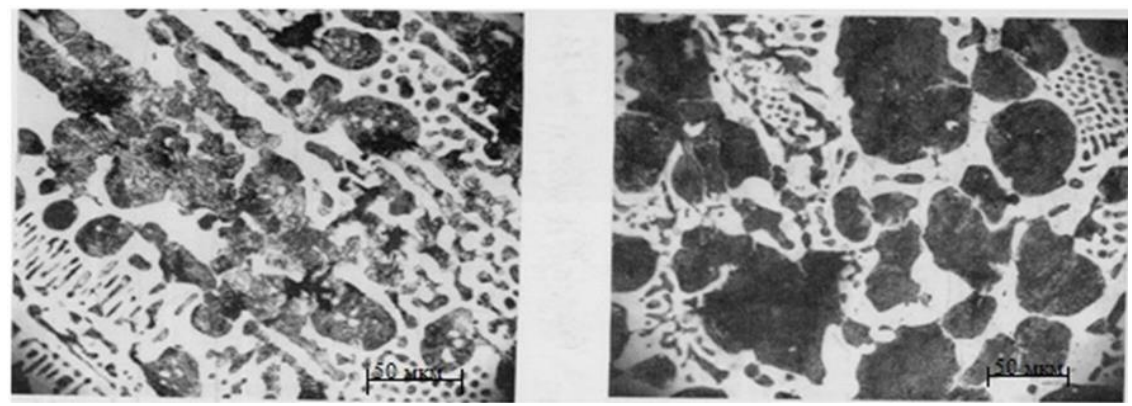
$\mathrm{a}$

$\mathrm{a}$ - hardening at $900{ }^{\circ} \mathrm{C} ; \mathrm{b}$ - hardening at $1050{ }^{\circ} \mathrm{C}$.

Fig. 4. Microstructure of gray cast iron (x300) after heat treatment.

It should be noted that there are no graphite inclusions in the structure of the hardened layer and the structural components obtained after heat treatment, electric spark and electric arc surfacing. Also, when studying the structure, no increase in grain size is observed, this is a favorable factor for the hardened layer properties.

With the help of electric arc surfacing, a layer of any thickness can be obtained; at the same time, with electric spark surfacing, the required thickness is obtained in several passes.

The electrodes were made of gray cast iron of the composition (\%): $\mathrm{C}-3.8 \ldots 4.2 \%, \mathrm{Si}-$ $0.75 \%, \mathrm{Mn}-0.24 \%, \mathrm{~S}-0.08 \%, \mathrm{P}-0.08 \%, \mathrm{Fe}-94.65 \ldots 95.05 \%$, they were subjected to heat treatment and mechanical properties presented in table 1.

Table 1. Influence of heat temperature for hardening on mechanical properties of gray cast iron.

\begin{tabular}{|l|c|c|c|}
\hline \multicolumn{1}{|c|}{ Heat treatment } & $\boldsymbol{\sigma}_{\mathbf{B}}, \mathbf{M P a}$ & $\boldsymbol{\delta}, \boldsymbol{\%}$ & $\boldsymbol{\Psi}, \boldsymbol{\%}$ \\
\hline without heating & 125 & 2,0 & 1 \\
\hline Hardening at $700^{\circ} \mathrm{C}$, cooling in water & 200 & 3,1 & 1 \\
\hline Hardening at $750^{\circ} \mathrm{C}$, cooling in water & 202 & 1,7 & 1 \\
\hline Hardening at $800^{\circ} \mathrm{C}$, cooling in water & 214 & 1,5 & 1 \\
\hline Hardening at $850^{\circ} \mathrm{C}$, cooling in water & 232 & 1,5 & 1 \\
\hline Hardening at $900^{\circ} \mathrm{C}$, cooling in water & 241 & 1,5 & 1 \\
\hline Hardening at $1000^{\circ} \mathrm{C}$, cooling in water & 240 & 1 & 1 \\
\hline Hardening at $1050^{\circ} \mathrm{C}$, cooling in water & 251 & 1 & 1 \\
\hline Annealing at $900^{\circ} \mathrm{C}$, exposure period 1 hour $(2$ cycles $)$ & 235 & 1 & 1 \\
\hline
\end{tabular}

Electric arc surfacing made it possible to obtain a composition "wear-resistant layer base metal" and the thickness of the hardened layer was sufficient for working in an abrasive environment, but the use of this method leads to strong heating of the near-weld zone and an increase in grain size (Fig. 5).

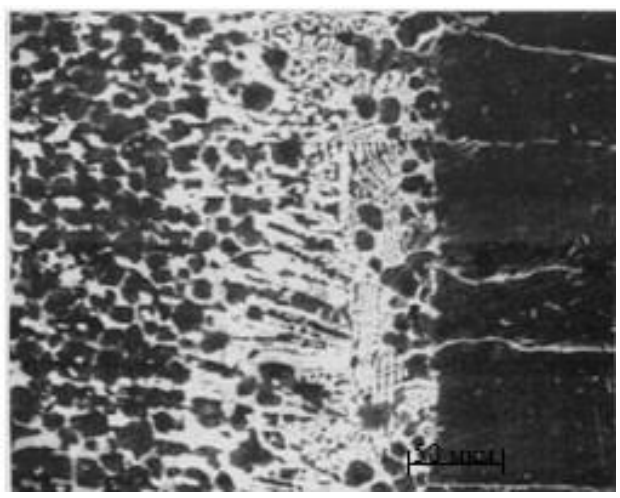

Fig. 5. Microstructure of the deposited layer after electric arc deposition (x80).

There were no graphite inclusions in the structure of the metal deposited by the electrospark method, which gives grounds for a conclusion about preservation of the 
structural components obtained after heat treatment and electrospark surfacing. Also, there was no increase in grain size in the deposited layer, which favorably affects its properties (Fig. 6).

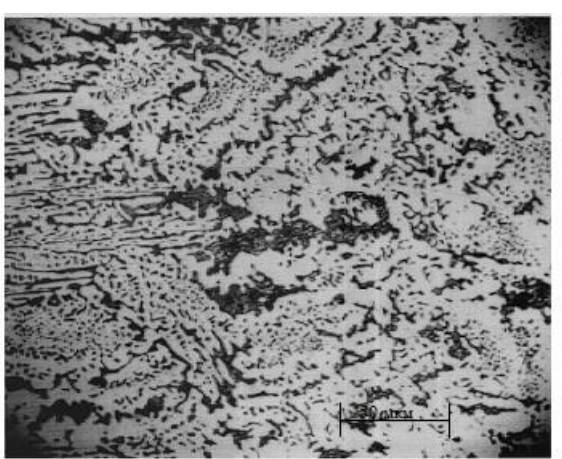

a

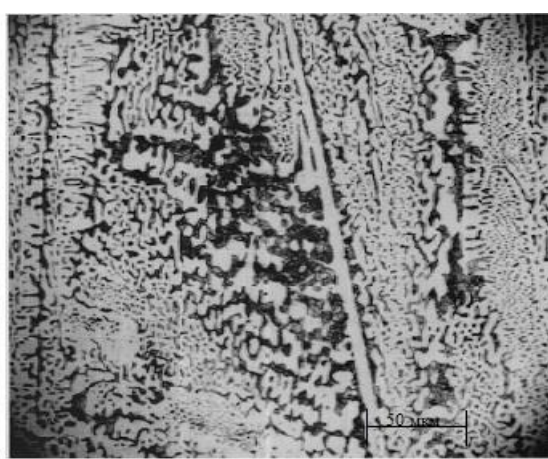

6

a - hardening at $900^{\circ} \mathrm{C}$, cooling in water; $\mathrm{b}$ - annealing at $900^{\circ} \mathrm{C}$, exposure period 1 hour 2 cycles (x300)

Fig. 6. Microstructure of gray cast iron after heat treatment.

Measurement of the hardened layer microhardness showed that the surface layer deposited by electrodes of gray cast iron, subjected to preliminary heat treatment in the form of quenching at a temperature of $900^{\circ} \mathrm{C}$ and subsequent cooling in water, has a higher hardness $(4800 \ldots 5000 \mathrm{MPa})$ than the base material, which demonstrates the value of this indicator of $2300 \ldots 2400 \mathrm{MPa}$. The optimum thickness of the deposited layer is at the level of $1.0 \ldots 2.5 \mathrm{~mm}$ (Fig. 5).

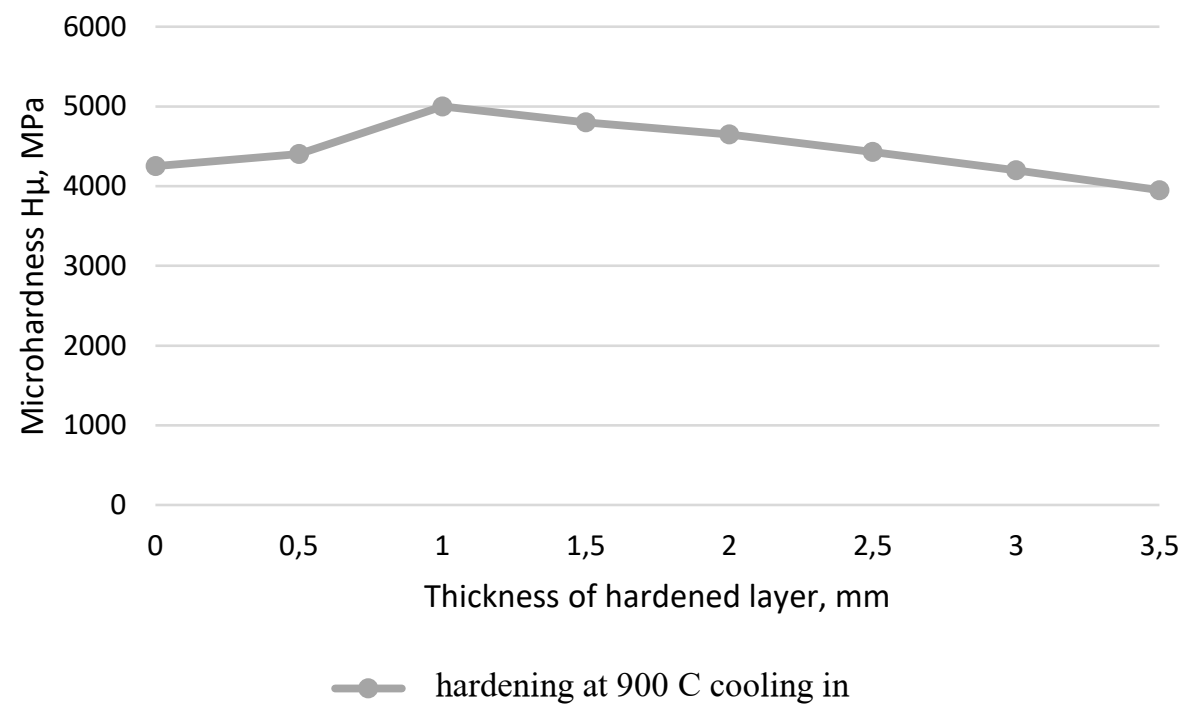

Fig. 7. Influence of heat treatment of electrodes on hardness change in the hardened layer over the cross section of the samples. 


\section{Conclusions}

Uneven wear of soil-cutting parts, observed during machine operation, requires a differentiated approach to the choice of technology for applying hardening of their friction surfaces. Based on the properties of the materials used for the manufacture of soil-cutting parts, gray cast iron, which has the best price-quality ratio of the finished product, is preferable for their strengthening. The technology of applying a hardened layer differentiated in thickness on the surface of the part requires the presence of maps of wear rates expected in subsequent operation. Obtaining such maps of abrasive wear can be carried out by preloading the soil-cutting parts. As a result, the differentiated application of the hardened layer makes it possible to save surfacing materials and subsequently achieve the required service life during operation.

The technology of electric arc hardening provides regulation of the thickness of the deposited layer and allows the use of surfacing oriented to the abrasive wear maps.

\section{References}

1. E.V. Sheina, L.O. Karpachevsky, Grif and K (2007)

2. I. M. Panov, V. I. Vetokhin, Phoenix (2008)

3. M. M. Severnev, N. N. Podlekarev, V. Sh. Sokhadze, V. O. Kitikov, Navuka (2011)

4. I. G. Goryacheva, Nauka (2001)

5. A.V. Chichinadze, E. D. Brown, N. A. Boucher, Mechanical Engineering (2001)

6. M. G. Kurbanova, A. P. Chernysh, O.V. Sankina, O.N. Byziyan, Achievements of science and technology of the agro-industrial complex, 5 (2013)

7. M.V. Chibryakov, O.V. Sankina, Bulletin of the International Academy of Agrarian Education, 39 (2018)

8. V. Shibe, V. Chawla, Transactions of the Indian Institute Of Metals, 9 (2018)

9. D. Martinazzi, V. B. Lemos Guilherme, M. Landell Renan, et al., Periodic tche chemistry, 31 (2019)

10. F. J. Carcel-Carrasco, M. Pascual-Guillamon, F. Salas-Vicente et al., Metals, 1 (2019)

11. U. Reisgen, R. Sharma, S. Wieland et al., Materials science and engineering, 12 (2018)

12. R. Winiczenko, M. Kaczorowski, A. Skibicki, J. of the Brazilian society of mechanical sciences and engineering, 7 (2018)

13. Y. Saraev, S. Gladkovsky, S. Lepikhin, I. Kamantsev, A. Lunev, M. Perovskaya, Metal processing - metal working andes material scene, 2 (2018)

14. M. Chamim, D. K. Triyono, Book Series: AIP Conference Proceedings, 1788 (2017)

15. P. Ambroza, S. Bockus, L. Kavaliauskiene, Archives Of Metallurgy And Materials, 2 (2013) 